\title{
Dexmedetomidine versus midazolam for sedation during endoscopy: A meta-analysis
}

\author{
FAN ZHANG ${ }^{1}$, HAO-RUI SUN ${ }^{1}$, ZE-BING ZHENG ${ }^{2}$, REN LIAO $^{1}$ and JIN LIU ${ }^{1}$ \\ ${ }^{1}$ Department of Anesthesiology and Translational Neuroscience Center, West China Hospital, Sichuan University, \\ Chengdu, Sichuan 610041; ${ }^{2}$ Department of Pediatrics, Zunyi Medical College, Zunyi, Guizhou 563000, P.R. China
}

Received December 3, 2014; Accepted February 2, 2016

DOI: $10.3892 /$ etm.2016.3186

\begin{abstract}
Patients undergoing endoscopy frequently require sedation, which commonly includes the administration of midazolam or dexmedetomidine. Previous meta-analyses have mainly focused on comparing the effects of these two drugs in intensive care unit patients. In the present study, randomized controlled trials (RCTs) that compared the sedative and clinical effectiveness of these two drugs in patients undergoing endoscopy were searched in a number of databases. The meta-analysis showed that dexmedetomidine demonstrated a significantly lower rate of respiratory depression and adverse events compared with those presented upon midazolam administration. A significant difference was also observed in the sedation potency of the sedatives. The current controlled data suggest that dexmedetomidine may be an alternative to midazolam in the sedation for endoscopy. However, more high-quality and well-designed studies are required to further evaluate this conclusion.
\end{abstract}

\section{Introduction}

Endoscopic procedures are of great importance for the diagnosis and treatment of various diseases, including upper gastrointestinal bleeding, esophageal dilatation and foreign body removal. However, anxiety, pain, fear and gastrointestinal reactions may cause patients to be less cooperative during endoscopy, and may even induce harmful cardiovascular adverse events (1); therefore, the role of sedation in endoscopy is significant. Higher doses of sedative drugs have been found to result in improved patient cooperation during the procedure and satisfaction $(2,3)$. Although various sedative agents are commonly used, the 'ideal' agent for endoscopy sedation remains to be established.

Correspondence to: Professor Jin Liu, Department of Anesthesiology and Translational Neuroscience Center, West China Hospital, Sichuan University, 37 Guo Xue Xiang, Chengdu, Sichuan 610041, P.R. China

E-mail: 292296517@qq.com

Key words: dexmedetomidine, midazolam, sedation, endoscopy, meta-analysis
Midazolam, the most common agent used for sedation, is a benzodiazepine with rapid onset of action and short duration of sedative effect (4). It produces central nervous system depression effects through the stimulation of $\gamma$-amino butyric acid receptors (5). To date, midazolam remains the predominant intensive care unit (ICU) sedative agent (6). However, it has certain undesirable side effects, such as delayed recovery of memory, long-term behavioral changes such as long-term cognitive dysfunction and respiratory depression.

Dexmedetomidine is a new-type, highly selective $\alpha_{2}$-adrenoceptor agonist, which has sedative, amnestic, sympatholytic and analgesic effects (7). It was first approved for use in ICU in 1999, and its use has been rapidly extended to various other clinical situations (8). A previous study has reported that dexmedetomidine may be a possible alternative to midazolam in sedation (9). As the use of dexmedetomidine has increased, associated adverse effects, such as hypotension and bradycardia, have been reported (10). Dexmedetomidine is increasingly used in the sedation of patients in different clinical situations.

Therefore, a meta-analysis was performed in the present study in order to compare the effects of the two drugs, midazolam and dexmedetomidine, in sedation during endoscopy by analyzing the most recently-published controlled trials.

\section{Materials and methods}

Result reliability. To ensure the reliability of the present meta-analysis, the results were reported according to the Preferred Reporting Items for Systematic Reviews and Meta-analyses statement (11).

Literature search strategy. The following digital databases were searched for the identification of studies: PubMed (www.ncbi. nlm.nih.gov/pubmed), Cochrane Library (www.cochranelibrary. com/), Ovid (ovidsp.ovid.com/autologin.cgi) and ClinicalTrails (https://clinicaltrials.gov/) databases. In addition, Chinese databases were searched, including CQVIP (http://en.cqvip. com/), WanFang Data (www.wanfangdata.com/) and Chinese Biomedical Literature databases (www.sinomed.ac.cn). All the databases were searched up to November 2014, with no language restriction. The literature search was performed using the relevant keywords of 'dexmedetomidine', 'midazolam', 
Table I. Patient characteristics in studies comparing the use of dexmedetomidine with midazolam for sedation during endoscopy.

\begin{tabular}{|c|c|c|c|c|c|c|}
\hline Authors & Year & Patients, $\mathrm{n}$ & Age, years & Male, $\mathrm{n}$ & Female, $\mathrm{n}$ & Refs. \\
\hline Wei et al & 2014 & $30 / 30$ & $35.3 / 36.6$ & $16 / 17$ & $14 / 13$ & (17) \\
\hline Sethi et al & 2014 & $30 / 30$ & $42 / 44$ & $13 / 14$ & $17 / 16$ & (18) \\
\hline Demiraran et al & 2007 & $25 / 25$ & $42.2 / 43.3$ & $13 / 9$ & $12 / 16$ & (19) \\
\hline Dere et al & 2010 & $30 / 30$ & $57.9 / 60.1$ & $22 / 21$ & $8 / 9$ & (20) \\
\hline Zhang et al & 2013 & $30 / 30$ & $\geq 65$ & NR & NR & $(21)$ \\
\hline Li et al & 2014 & $30 / 30$ & 55.1 & 38 & 22 & (22) \\
\hline Arpaci and Bozkirli & 2013 & $20 / 20$ & $50.4 / 47.1$ & $6 / 7$ & $14 / 13$ & (23) \\
\hline Karaaslan et al & 2007 & $35 / 35$ & $32.5 / 34.4$ & $23 / 21$ & $12 / 14$ & (24) \\
\hline Liao et al & 2012 & $99 / 98$ & $58.5 / 60.1$ & $61 / 62$ & $38 / 36$ & $(25)$ \\
\hline
\end{tabular}

NR, not reported.

Table II. Main results of the meta-analysis.

\begin{tabular}{lccc}
\hline & \multicolumn{2}{c}{ Patients included } & \\
\cline { 2 - 4 } Results & Dexmedtomidine & Midazolam & $\begin{array}{c}\text { Standard deviation } \\
\text { Mean Difference }\end{array}$ \\
\hline $\mathrm{SpO}_{2}$ & 224 & 223 & $1.25[-0.31,0.61]$ \\
$\mathrm{MAP}$ & 175 & 175 & $-0.08[-0.29,0.1]$ \\
$\mathrm{RSS}$ & 95 & 95 & $0.64[0.35,0.93]$ \\
Adverse events & & & - \\
Hypertension & 4 & 12 & - \\
Hypotension & 11 & 8 & - \\
Tachycardia & 13 & 12 & - \\
Hypoxia & 1 & 11 & - \\
Nausea & 10 & 2 & - \\
Other & 31 & 69 & 0.49 \\
Total & 70 & 112 & 0.40001 \\
\hline
\end{tabular}

MAP, mean arterial pressure; RSS,. Ramsay sedation scale.

'Dormicum', 'endoscopy'. For the Chinese databases, free-text terms were used, including 'mi da zuo lun' or 'mi zuo an ding' (which is the translation of 'midazolam' in Chinese), as well as 'nei jing', 'chang jing' and 'wei jing' (which refer to different types of endoscopy in Chinese). The search strategy was independently performed by two investigators. Any disagreements were resolved by consensus and discussion.

Study selection. The inclusion criteria for the trials included in the present meta-analysis were as follows: i) Randomized controlled trials (RCTs); ii) the study focused on the sedation effects of dexmedetomidine and midazolam; and iii) the study involved patients with an American Society of Anesthesiologists (ASA) (12) grade I to III, and who presented for outpatient endoscopy procedures under conscious sedation. Exclusion criteria were as follows: i) Case reports, letters, reviews, editorial articles, meta-analyses and retrospective studies; ii) duplicates of previous published articles; and iii) studies which included children.

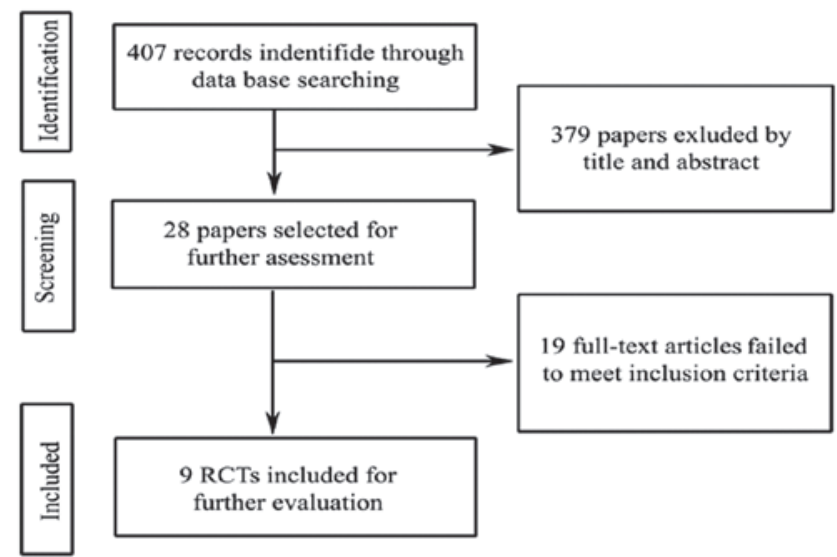

Figure 1. Study selection flow chart. RCT, randomized controlled trial.

Data extraction. The following variables were recorded for each of the studies: First author, journal, publication date, 
A

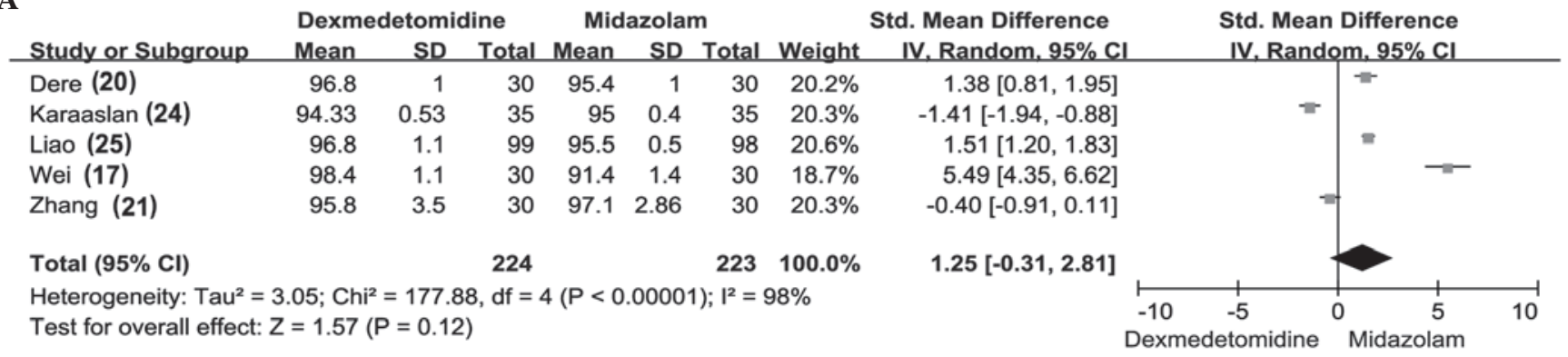

B

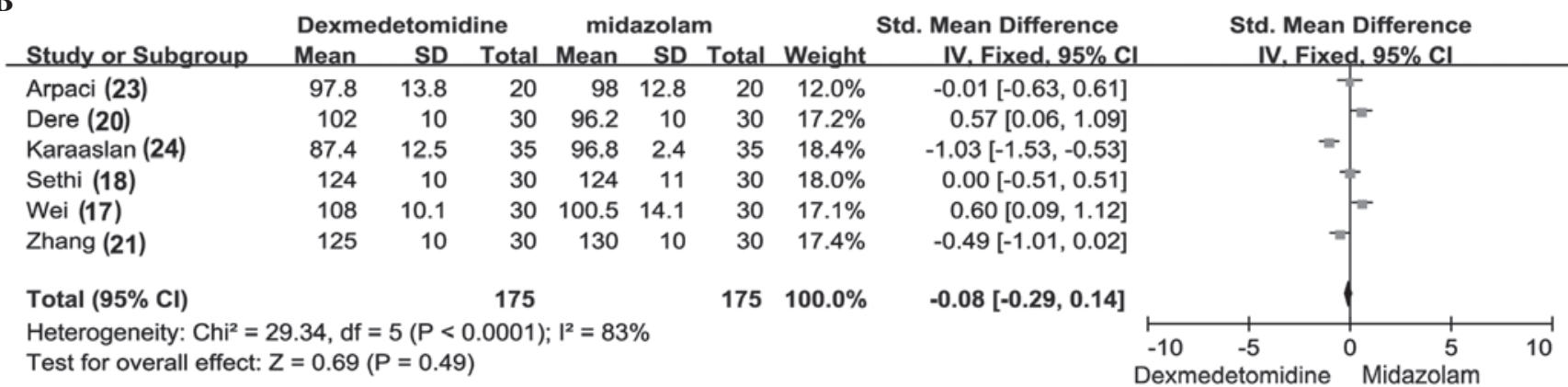

Figure 2. Forest plots of the dexmedetomidine sedation compared with midazolam in terms of (A) peripheral oxygen saturation, and (B) mean arterial pressure. $\mathrm{SD}$, standard deviation; Std, standard; CI, confidence interval.

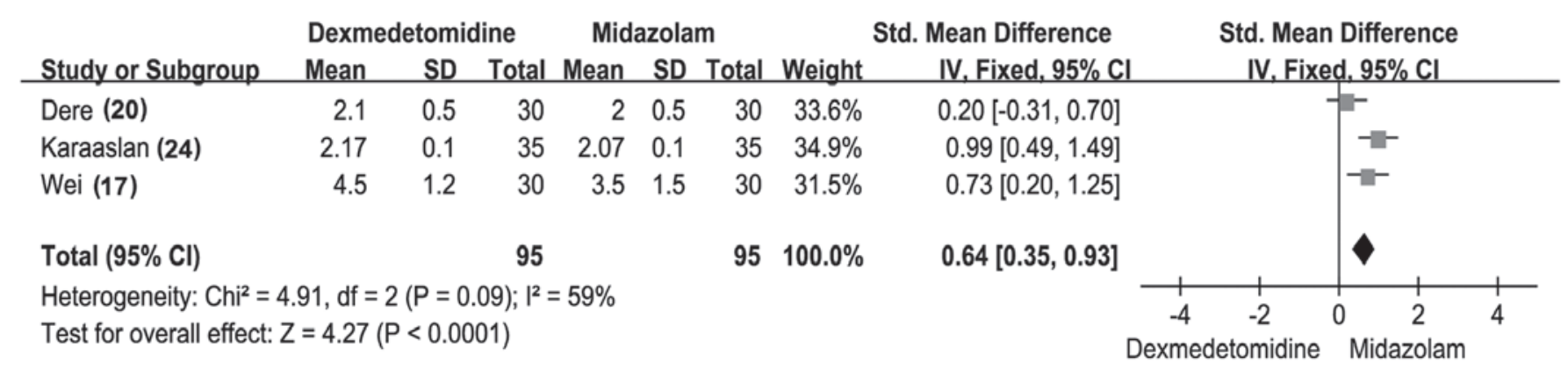

Figure 3. Forest plot comparing dexmedetomidine with midazolam in endoscopy based on the recorded Ramsay sedation scale scores of patients. SD, standard deviation; Std, standard; 95\% CI, 95\% confidence interval.

country, baseline difference, method of randomization, degree of blinding, dropouts and withdrawals. By baseline difference we mean the basic health condition of the patients. The degree of blinding means the degree of blinding method, i.e. double or single-blinding used. Finally the withdrawals indicate the patients who did not finish the study. The primary outcome of interest were changes in vital signs, including the continuous peripheral oxygen saturation $\left(\mathrm{SpO}_{2}\right)$, heart rate, respiration rate, mean arterial pressure (MAP) of the patients, Ramsay sedation scale (RSS) (13) and Alertness/Sedation scale $(\mathrm{OOA} / \mathrm{S})(14)$. Secondary outcomes included numeric rating scale pain scores, post-procedure satisfaction questionnaire and adverse events.

Statistical analysis. All statistical analyses were performed using Review Manager version 5.2 statistical software (Cochrane Collaboration, Copenhagen, Denmark). Dichotomous data are expressed as odds ratios (ORs) with 95\% confidence intervals (CIs). Continuous variables are presented as the standard mean difference (SMD). The statistical significance of the pooled value was evaluated using the $\mathrm{Z}$ test, while heterogeneity was analyzed via the $\mathrm{I}^{2}$ test. Where the heterogeneity test showed no heterogeneity, the data were processed via a fixed effects model; otherwise, a random effects model was conducted for the analysis. Begg's funnel plots were used to detect publication biases. $\mathrm{P}<0.05$ was considered to indicate a statistically significant difference in all the analyses. To ensure the accuracy of the outcomes, two researchers assessed the data independently and obtained the same results.

Assessment of study quality. The included studies were reviewed and assessed for methodological quality using the Jadad composite scale (15). High-quality trials scored $>3$ out of a maximum score of 5 (16).

\section{Results}

Study characteristics. The process of study selection is shown in Fig. 1. According to the inclusion criteria, 9 studies 


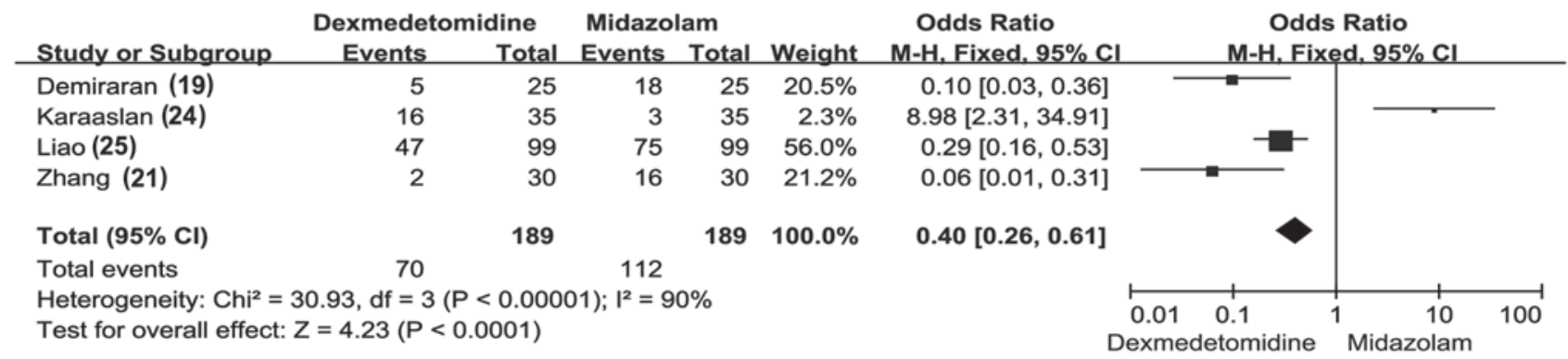

Figure 4. Forest plot comparing dexmedetomidine with midazolam in endoscopy based on the number of adverse events reported. CI, confidence interval.

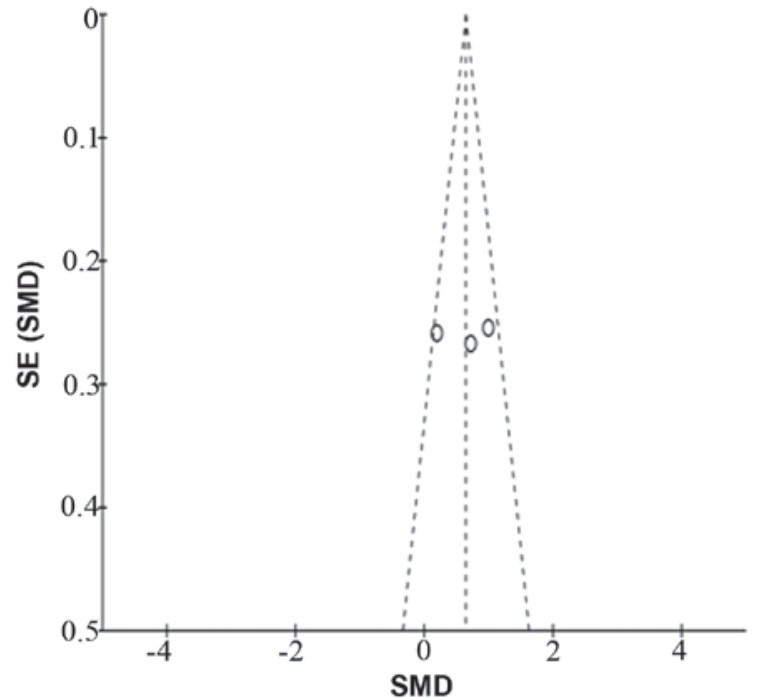

Figure 5. Funnel plot of the Ramsay sedation scale of dexmedetomidine and midazolam. SE, standard error; SMD, standard mean difference.

were included in the present meta-analysis. The included studies were published as full text between January 2007 and December 2014. Of the 657 patients included in the 9 eligible RCTs (17-25), 329 patients were allocated to the dexmedetomidine group, while 328 patients comprised the midazolam group. The sedation effects in the two groups were evaluated. Patient characteristics, including age and gender, are shown in Table I.

Quality of the included studies. The mean Jadad score of the included studies was 3 out of a maximum possible score of 5 . This indicated that the majority of the included RCTs were of moderate quality, although 2 Chinese trials $(21,22)$ were of low quality (Jadad score of <3), which was a result of relatively poor study design.

\section{Trial outcomes.}

$\mathrm{SpO}_{2}$. Statistical analysis performed in 5 of the eligible studies $(17,20,21,24,25)$ revealed that the $\mathrm{SpO}_{2}$ showed no statistically significant difference between the dexmedetomidine and midazolam groups (Fig. 2A). These 5 studies were used for the analysis because of their similar study index which made them comparable for a meta-analysis. In addition, there was no evidence of significant heterogeneity (SMD, 1.25; 95\% CI, -0.31 to $2.81 ; \mathrm{P}=0.12$; Fig. 2A).
MAP. In total, 6 studies reported the MAP of patients following administration of the two agents $(17,18,20,21,23,24)$. The results demonstrated that there was no significant difference in the MAPs between the dexmedetomidine and midazolam groups [SMD, $-0.08 ; 95 \% \mathrm{CI},-0.29$ to 0.14$]$. In addition, no significant heterogeneity was detected (Fig. 2B).

$R S S$. In total, 3 of the 9 included studies reported the RSS of patients $(17,20,24)$. The results revealed that the RSS scores of patients administered dexmedetomidine were significantly higher compared with those in the midazolam group patients (SMD, 0.64; 95\% CI, 0.35-0.93; P<0.0001). However, no significant heterogeneity was detected in the results (Fig. 3). With regard to normal adults, $0.05-0.075 \mathrm{mg} / \mathrm{kg}$ midazolam intravenous injection produces the effect of sedation. A higher dose of $0.1-0.15 \mathrm{mg} / \mathrm{kg}$ is commonly used for anaesthesia induction. For dexmedetomidine, a continuous intravenous infusion of $0.2-0.7 \mu \mathrm{g} / \mathrm{kg} / \mathrm{h}$ can achieve a good sedation outcome. General anaesthesia stages may be induced above such a drug dose.

Adverse events. Out of the 9 eligible studies, 4 trials (involving 189 patients) compared the adverse events reported in patients administered with dexmedetomidine and midazolam $(19,21,24,25)$. The main adverse events that were reported were respiratory depression, nausea and vomiting, dysphoria, reflux, dizziness, abdominal distention and pain.

The pooled results displayed a statistically significant difference between the subgroups (Fig. 4). In the dexmedetomidine group, a significantly lower number of adverse events were reported, compared with the midazolam group (OR, $0.40 ; 95 \%$ CI, 0.26-0.61; P<0.0001; Fig. 4). The adverse events and main trail results were shown in Table II.

Testing for publication bias. A funnel plot of the outcome of the RSS score following sedation with dexmedetomidine and midazolam in the included studies demonstrated there was no significant publication bias (Fig. 5). However, the number of trials included was $<10$, thus this conclusion may not be entirely accurate.

\section{Discussion}

Endoscopy is an essential procedure for clinical diagnosis and treatment of various diseases such as gastrointestinal neuroendocrine tumours (26-29), gastroesophageal reflux , lung cancer and cervical cancer. Different endosocopes may be used to diagnose different diseases. For example, cystoscopy is used to diagnose diseases in the urinary system. Although conscious 
sedation is the most widely used method to relieve sickness and pain during endoscopy, the sedation agents used greatly vary among different regions (30). Midazolam has long been regarded as the golden standard for sedation. However, dexmedetomidine has been increasingly studied in recent years.

To the best of our knowledge, Adams et al (31) performed the first meta-analysis concerning the effects of dexmedetomidine and midazolam in adult ICU patients. In addition, Sun et al (32) performed a systemic review regarding premedication in children using the two drugs. The present meta-analysis evaluated the sedation effects of dexmedetomidine and midazolam during endoscopy.

The results of the current meta-analysis suggested that dexmedetomidine sedation may achieve a more stable respiratory system. Patients sedated with dexmedetomidine had statistically lower hypoxia rates in the present study. Furthermore, patients administered dexmedetomidine have been previously shown to be easily awaken during endoscopy and experience less respiratory depression compared with those sedated with midazolam $(33,34)$. Therefore, dexmedetomidine may be advantageous compared with midazolam for the sedation of patients with previous history of respiratory diseases.

As reported previously (35), the most significant complications associated with dexmedetomidine are hypotension and bradycardia. However, the results of the present meta-analysis challenge these conclusions, since no significant differences were observed in the arterial pressure or heart rate of the 175 patients investigated. This is in agreement with the results of Yu et al (36) reporting that the SBP values did not present any differences after slow infusion of the drug over a $10 \mathrm{~min}$ time-frame. Instead, appropriate dose and transfusion velocity of dexmedetomidine may achieve favorable cardiovascular stability $(37,38)$. However, dexmedetomidine should be used with caution in patients diagnosed with severe sinus bradycardia or heart block (39).

Potent sedation effects may be the main advantage of dexmedetomidine for endoscopy. The present study results revealed that the RSS was significantly higher in the dexmedetomidine group, while the OOA/S of patients in the dexmedetomidine was also higher compared with that in the midazolam group. Even a maintenance dose of $0.5 \mu \mathrm{gkg} / \mathrm{h}$ dexmedetomidine was able to provide better sedation compared with $0.05 \mathrm{mgkg}^{-1}$ midazolam. This indicated a superior sedation effect of dexmedetomidine compared with midazolam (40-42). However, despite its outstanding sedation potency, it is also able to maintain awarenesss at the sedative dose $(43,44)$, which may enable the patient to change positions according to orders and make the endoscopy a more smooth procedure (45).

There was also a significant difference between the dexmedetomidine and midazolam groups in terms of the number of adverse events reported. Major complications, including hypertension, requirement for mandible support and intubation, were mainly observed in the midazolam group $(46,47)$. Therefore, dexmedetomidine may be advantageous with regard to the prognosis and outcome of endoscopy patients.

Several limitations exist in the present meta-analysis. First, two of the included trials were published in Chinese $(21,22)$, and these studies were of relatively poor quality due to unclear concealment of research details. In addition, it was difficult to draw a definitive conclusion regarding whether dexmedetomidine was a better sedative compared with midazolam since no uniform criteria exist to assess the effects of sedatives. Furthermore, a greater number of well-designed trials are required to confirm the aforementioned results.

In conclusion, the present meta-analysis included 9 RCTs reporting sedation during endoscopic procedures, and the results indicated that the sedation effects of dexmedetomidine and midazolam were comparable in patients undergoing endoscopy. Therefore, the present study recommends that both of the medications should be considered for patient sedation during endoscopy.

\section{Acknowledgements}

This study was supported by a grant from the National Special grant projects of China (grant no. 201002005).

\section{References}

1. Eqer EI, White PF and Boqetz MS: Clinical and economic factors important to anaesthetic choice for day-case surgery. Pharmacoeconomics 17: 245-262, 2000.

2. Moon SH: Sedation regimens for gastrointestinal endoscopy. Clin Endosc 47: 135-140, 2014.

3. Kim KH: Safe sedation and hypnosis using dexmedetomidine for minimally invasive spine surgery in a prone position. Korean J Pain 27: 313-320, 2014.

4. Triantafillidis JK, Merikas E, Nikolakis D and Papalois AE: Sedation in gastrointestinal endoscopy: Current issues. World J Gastroenterol 19: 463-481, 2013.

5. Yilaz E, Hough KA, Gebhart GF, Williams BA and Gold MS: Mechanisms underlying midazolam-induced peripheral nerve block and neurotoxicity. Reg Anesth Pain Med 39: 525-533, 2014.

6. Chawla R, Myatra SN, Ramakrishnan N, Todi S, Kansal S and Dash SK: Current practices of mobilization, analgesia, relaxants and sedation in Indian ICUs: A survey conducted by the Indian society of critical care medicine. Indian J Crit Care Med 18: 575-584, 2014

7. Bajwa S and Kulshrestha A: Dexmedetomidine: An adjuvant making large lnroads into clinical practice. Ann Med Health Sci Res 3: 475-483, 2013.

8. Takrouri MS, Seraj MA, Channa AB, el-Dawlatly AA, Thallage A, Riad W and Khalaf M: Dexmedetomidine in intensive care unit: A study of hemodynamic changes. Middle East J Anesthesiol 16: 587-595, 2002.

9. Ihmsen H and Saari Ti: Dexmdetomidine. pharmacokinetics and pharmacodynamics. Anaesthesist 61: 1059-1066, 2012 (In German).

10. Bharati S, Pal A, Biswas C and Biswas R: Incidence of cardiac arrest increases with the indiscriminate use of dex medetomidine: A case series and review of published case reports. Acta Anaesthesiol Taiwan 49: 165-167, 2011.

11. Moher D, Liverati A, Tetalaff J, Altman DG; PRISMA Group: Preferred reporting items for systematic reviews and meta-analyses: The PRISMA statement. Ann Intern Med 151: 264-269, 2009.

12. Sankar A, Johnson SR, Beattie WS, Tait G and Wijeysundera DN. Reliability of the American society of anesthesiologists physical status scale in clinical practice. Br J Anaesth 3: 424-432, 2014.

13. Dawson R, von Fintel N and Nairn S: Sedation assessment using the Ramsay scale. Emerg Nurse 3: 18-20, 2010.

14. Zhan-Ying G, Chang-Ming W, Shuai T, Lin-Lin T and Yu-Feng H: Comparison of effects of different doses of dexmedetomidine on inhibiting tracheal intubation-evoked haemodynamic responce in the elderly patients. J Clin Diangn Res 9: 10-13, 2015.

15. Yang Z, Zheng Q and Wang Z: Meta-analysis for nasogastric or nasojejunal decompression after gastrectomy for gastric cancer. Br J Surg 95: 809-816, 2008.

16. Moher D, Pham B, Jones A, Cook DJ, Jadad AR, Moher M, Tugwell P and Klassen TP: Does quality of reports of randomized trials affect estimates of intervention efficacy reported in meta-analyses? Lancet 352: 609-613, 1998. 
17. Wei W, Chen Q, Zhang LC and Chen WH: Dexmedetomidine verses midazolam for sedation in upper gastrointestinal endoscopy. J Int Med Res 42: 516-522, 2014

18. Sethi P, Mohammed S, Bhatia PK and Gupta N: Dexmedetomidine verses midazolam for conscious sedation in endoscopic retrograde cholangiopancreatography: An open-lable randomized controlled trial. Indian J Anaesth 58: 18-24, 2014.

19. Demiraran Y, Korkut E, Tamer A, Yorulmaz I, Kocaman B, Sezen G and Akcan Y: The comparison of dexmedetomidine and miazolam used for sedation of patients during upper endoscopy: A prospective, randomized study. Can J Gastroenterol 21: 25-29, 2007.

20. Dere K, Sucullu I, Budak ET, Yeyen S, Filiz AI, Ozkan S and Dagli G: A comparison of dexmedetomidine versus midazolam for sedation, pain and hemodynamic control, during colonoscopy under conscious sedation. Eur J Anaesthesiol 27: 648-652, 2010.

21. Zhang G, Zheng FL, Ouyang W and Xiao DH: Small dose of dexmedetomidine in elderly patients for conscious sedation undergoing colonoscopy. Zhong Guo Nei Jing Za Zhi 19: 685-688, 2013 (In Chinese).

22. Li YX, Qu XH, Li HY, Luo ZH, Cong S, Liu B and Cui XG: The comparison of sedation with dexmedetomidine and midazolam used for enteroscopy. Xian Dai Sheng Wu Yi Xue Jin Zhan 14: 2293-2225, 2014 (In Chinese).

23. Arpaci AH and Bozkirli F: comparison of sedation effectiveness of remifentanil-dexmedetomidine and remifentanil-midazolam combinations and their effects on postoperative cognitive functions in cstoscopies: A randomized clinical trial. J Res Med Sci 18 107-114, 2013

24. Karaaslan K, Yilmaz F, Gulcu N, Colak C, Sereflican M and Kocoglu H: Comparison of dexmedetomidine and midazolam for monitored anesthesia care combined with tramadol via patient-controlled analgesia in endoscopic nasal surgery: A prospective, randomized, double-blind, clinical study. Curr Ther Res Clin Exp 68: 69-81, 2007.

25. Liao W, Ma G, Su QG, Fang Y, Gu BC and Zou XM: Dexmedetomidine versus midazolam for conscious sedaton in postoperative patients undergoing flexible bronchoscopy: A randomized study. J Int Med Res 40: 1371-1380, 2012.

26. Koca T, Dereci S, Karaham N and Akcam M. Gastrointestinal neuroendocrine tumors in two children. Indian Pediatr 1: 70-72, 2016.

27. Kenshi Yao. The endoscopic diagnosis of early gastric cancer. Ann Gastroenterol 1: 11-22, 2013.

28. Yu H, Yang AM, Lu WX, Zhou WX, Yao F, Fei GJ, Guo T, Yao LQ, He LP and Wang BM: Magnifying narrow-band imaging endoscopy is superior in diagnosis of early gastric cancer. World J Gastroenterol 30: 9156-9162, 2015.

29. Wang D, Wei XE, Yan L, Zhang YZ and Li WB. Enhanced CT and CT virtual endoscopy in diagnosis of heterotopic pancreas. World J Gastroenterol 33: 3850-3855, 2011.

30. Bell GD: Premedication, preparation and surveillance. Endoscopy 34: 2-12, 2002.

31. Adams R, Brown GT, Davidson M, Fisher E, Mathisen J, Thomson G and Webster NR: Efficacy of dexmedetomidine compared with midazolam for sedation in adult intensive care patients: A systematic review. Br J Anaesth 111: 703-710, 2013.

32. Sun $Y$, Lu Y, Huang $Y$ and Jiang H: Is dexmedetomidine superior to midazolam as a premedication in children? A meta-analysis of randomized controlled trials. Paediatr Anaesth 24: 863-874, 2014.
33. Tellor BR, Arnold HM, Micek ST and Kollef MH: Occurrence and predictors of dexmedetomidine infusion intolerance and failure. Hosp Pract (1985) 40: 186-192, 2012.

34. Huang Z, Chen YS, Yang ZL and Liu JY: Dexmedetomidine versus midazolam for the sedation of patients with non-invasive ventilation failure. Intern Med 51: 2299-2305, 2012.

35. Jalowiecki P, Rudner R, Gonciarz M, Kawecki P, Petelenz M and Dziurdzik P: Sole use of dexmedetomidine has limited utility for conscious sedation during outpatient colonoscopy. Anesthesiology 103: 269-273, 2005.

36. Yu C, Li S, Deng F, Yao Y and Qian L: Comparison of dexmedetomidine/fentanyl with midazolam/fentanyl combination for sedation and analgesia during tooth extraction. Int J Oral Maxillofac Surg 43: 1148-1113, 2014.

37. Schafrath E, Kuhlen R and Tonner PH: Analgesia and sedation in intensisve care medicine. Anaesthesist 53: 1111-1130, 2004 (In German).

38. Cheung CW, Ying CL, Chiu WK, Wong GT, Ng KF and Irwin MG: A comparison of dexmedetomidine and midazolam for sedation in third molar surgery. Anaesthesia 62: 1132-1138, 2007.

39. Arain SR and Ebert TJ: The efficacy, side effects and recovery characteristics of dexmedetomidine versus propofol when used for intraoperative sedation. Anesth Analg 95: 461-466, 2002.

40. Snapir A, Posti J, Kentala E, Koskenvuo J, Sundell J, Tuunanen H, Hakala K, Scheinin H, Knuuti J and Scheinin M: Effects of low and high plasma concentrations of dexmedetomidine on myocardial perfusion and cardiac function in healthy male subjects. Anesthesiology 105: 902-910, 2006.

41. Nelson LE, Lu J, Guo T, Saper CB, Franks NP and Maze M: The a2-adrenoceptor agonist dexmedetomidine converges on an endogenous sleep promoting pathway to exert its sedative effects. Anesthesiology 98: 428-436, 2003.

42. Prielipo RC, Wall MH, Tobin JR, Groban L, Cannon MA, Fahey FH, Gage HD, Stump DA, James RL, Bennett J and Butterworth $\mathrm{J}$ : Dexmedetomidine induced sedation in volunteers decreases regional and global cerebral blood flow. Anesth Analg 95: 1052-1059, 2002.

43. Gerlach AT, Dasta J, Armen S, Smith J, Steinberg S, Martin L and Cook C: Titration protocol reduces hypotension during dexmedetomidine infusion in critically ill surgical patients (abstract). Crit Care Med 34: A148, 2006.

44. Mack PF, Perrine K, Kobylarz E, Schwartz TH and Lien CA: Dexmedetomidine and neurocognitive testing in awake craniotomy. J Neurosurg Anesthesiol 16: 20-25, 2004.

45. Wijeysundera DN, Bender JS and Beattie WS: Alpha-2 adrenergic agonists for the prevention of cardiac complications among patients undergoing surgery. Cochrane Database Syst Rev 7: CD004126, 2009.

46. Coull JT, Jones ME, Ecan TD, Frith CD and Maze M: Attentional effects of noradrenaline vary with arousal level: Slective activation of thalamic pulvinar in humans. Neuroimage 22: 315-322, 2004

47. Menda FK, Köner O, Sayin M, Türe H, Imer P and Aykaç B: Dexmedetomidine as an adjunct to anesthetic induction to attenuate hemodynamic response to endotracheal intubation in patients undergoing fast-track GABG. Ann Card Anaesth 3: $16-21,2010$ 